Chapter 14

\title{
Quality of Life Issues of Patients with Multiple Myeloma
}

\author{
Klára Gadó and Gyula Domján \\ Additional information is available at the end of the chapter \\ http://dx.doi.org/10.5772/55625
}

\section{Introduction}

The great advance in the field of anti-myeloma therapy in the last few decades has resulted in a huge improvement of overall and disease-free survival. Nevertheless, multiple myeloma $(\mathrm{MM})$ is still an incurable disease.

There are two issues emerging. On one hand, the patient lives together with the illness for a long time, and on the other hand, the thought of incurable illness hangs over their head like the sword of Damocles for a longer time. Quality of life (QoL) issues are coming into focus because of the longer survival times.

Problems related to the disease such as pain, fatigue, bone fracture-induced inconveniences, complications such as infections, neuropathy, thrombosis, osteonecrosis of the jaw, mucositis, as well as invasive interventions emphasize the importance of supportive care.

The social and economic environment of the patients, their participation in the world of labor, financial resources, changes in their family and in their circle of friends all have a great impact on the QoL of patients.

The stigmata of chronic illness and malignancy also contribute to the development of depression thus influencing quality of life. At the last stage of life it is a very hard task for the patient to face dying.

At the same time, family members are also in a troublesome situation. To accept the incurable illness of a beloved member of the family is a great psychical burden. Beside these, the increase of physical burden may cause insoluble task for the folks and this may generate sense of guilt.

Nowadays, the measuring of QoL is in a class by itself. QoL has become a prognostic factor. Several studies have demonstrated that better quality of life goes hand in hand with better prognosis. This is also the case with multiple myeloma. 
Results of examinations of QoL may help us to provide professional and effective support to the patient and their family through a holistic approach. Multidisciplinary co-operation is essential.

\section{Main features of Multiple Myeloma}

Multiple myeloma is the second most common hematological cancer and represents $10 \%$ of all hematological malignancies and $1 \%$ of all cancers. The annual incidence of the disease in the US is 4 in 100,000. Approximately 100,000 new cases of MM are diagnosed each year worldwide [1]. MM accounts for $1 \%$ of all cancer-related deaths (approximately 72,000 deaths annually). The vast majority of the patients diagnosed with MM are 70-80 years old. MM is characterized by unregulated plasma cell proliferation in the bone marrow. These malignant plasma cells produce and secrete abnormal immunoglobulin (Ig) or immunoglobulin fragments. The monoclonal $\mathrm{lg}$ in the sera can cause hyperviscosity and this is one of the major symptoms of the disease. Clinical features and typical laboratory findings of MM include fatigue, bone pain, osteolythic bone lesions, pathologic bone fracture, anemia, hypercalcaemia, renal insufficiency, elevation of monoclonal $\mathrm{Ig}$ in the sera and/or in the urine and elevated erythrocyte sedimentation rate. The etiology of $\mathrm{MM}$ is unknown but aside from several environmental factors that are suspected, more and more cytogenetic alterations involved with the oncogenic process are detected $[2,3]$.

\section{The aims of MM treatment}

Despite the huge advance in the field of MM treatment, the disease has still remained incurable.

The main goal of treatment is the prolongation of survival. By the 1980's to 1990's, the survival of untreated patients had increased from mere months to 3-5 years. The introduction of intensive treatment, such as high-dose chemotherapy with autologous stem cell transplantation (ASCT), further prolonged the overall survival. Novel agents, including immunomodulatory drugs, such as thalidomide and lenalidomide, and the proteosome inhibitor bortezomib have dramatically changed the results in the past decade. Besides overall survival, disease-free survival has also been prolonged and the life expectancies of refractory and relapsed patients are also largely improved [4].

The only curative treatment option is allogeneic stem cell transplantation due to antitumor immunity mediated by donor lymphocytes. However, morbidity and mortality related to graft-versus-host disease remain a challenge and regarding the average age of MM patients it remains an option for only a minority of patients.

Depending on stage of the disease, median survival varies between 5-10 years for patients with ISS stage I disease undergoing stem-cell transplant and/or receiving novel anti-myeloma regimens [5]. However, outcomes have typically been poor for patients with high-risk disease and despite recent therapeutic advances the outlook for such patients remains unfavorable [6]. 


\section{Consequences of MM being a disease of the elderly}

The incidence of multiple myeloma (MM) increases with age and with the aging of the population, the number of adults with $\mathrm{MM}$ is expected to double in the next 20 years. Intensification of anti-myeloma therapy has resulted in a huge prolongation of survival data but this data mainly refers to younger patients who are eligible for these treatment modalities.

Older patients are ineligible for high-dose therapy because it causes an unacceptably high mortality rate in that patient population. Several co-morbidities of this setting or poor performance status prevent the success of intensive treatment.

On the other hand the significance of supportive measures for these patients has become a greater value. Besides the extended duration of survival, to improve the quality of survival by alleviating symptoms and achieving disease control while minimizing the adverse effects of the treatment has become a major goal [7].

Factors affecting prognosis include burden of disease, type of cytogenetic abnormality present, patient related factors (such as age and performance status) and treatment response factors.

Asymptomatic myeloma (smoldering myeloma) does not require any treatment, only observation (watch and wait) is needed.

The choice of first-line treatment depends on a combination of factors.

For patients under 70 and with good performance status, the treatment of choice is high-dose chemotherapy with ASCT.

The majority of patients are transplantation-ineligible because of poor performance status or co-morbidities. These patients are therefore offered a less intensive single-agent or combination chemotherapy. Typically, combination therapies include chemotherapy with an alkylating agent and corticosteroids. More recent treatment options may also include combination therapies that incorporate drugs such as thalidomide, bortezomib and lenalidomide [8].

Regarding themaintenancetherapy, if completeremission(CR)hasbeen reached there isnoneed for maintenance therapy with thalidomide or lenalidomide because there is no significant differencein OS. In the case of lenalidomide, a significantly increased risk of secondary malignancies was reported [9]. Maintenanceisadvised for patients whohavenotreachedCR. In thesecases, one of the new drugs (thalidomide, lenalidomide or bortezomib) is the drug of choice [10].

However, in line with all these improvements in the field of chemotherapy, some new questions have emerged. The patient has gained a longer life, but is this life good enough? Is it worth the sea of difficulties during the treatment period and even afterward? To answer these questions, QoL measurements can offer valuable meaning.

\section{Definition of quality of life and importance of QoL measuring}

QoL can be defined in many ways. As a general term it is used to indicate the well-being of people and societies. A person's environment, physical and mental health, education, recreation, social well-being, freedom, human rights and happiness are also significant factors. 
The World Health Organization (WHO) defines QoL as individuals' perception of their position in life in the context of the culture and value systems in which they live and in relation to their goals, expectations, standards, and concerns [11].

As illness and its treatment affect the psychological, social, and economic well-being, as well as the biological integrity of individuals, any definition should be all encompassing while allowing individual components to be delineated. This allows the impact of different disease states or interventions on overall or specific aspects of QoL to be determined.

QoL is measured in a variety of contexts. Aside from healthcare, it is also used in international development and political science. This results in diverse definitions being given to the term. Factors that are considered are both qualitative and quantitative. Many local, national and international organizations conduct surveys and psychological tests to determine an individual or society's life quality for different purposes.

A major rule for physicians is the principle of "nil nocere". While making an effort to reach better and better disease control for cancer patients, we often neglect the repercussions of the patient in regards to "being ill", to the consequences of the treatment, and to the disease per se. The main purpose for all clinicians is therefore to improve the quality of the patient's life and to avoid iatrogenic harm. It is not enough to make implicit, subjective judgments about QoL when treating a patient. Making explicit, objective assessments about QoL using validated tools and instruments is needed. Formal assessment of QoL is now a mandatory requirement in most clinical trials.

\section{Health-Related Quality of Life (HRQoL)}

WHO defines health as "A state of complete physical, mental, and social well-being not merely the absence of disease." The measurement of health and the effects of health care must include not only an indication of changes in the frequency and severity of diseases but also an estimation of well being and this can be assessed by measuring the improvement in the QoL related to health care [11].

HRQoL can be defined as self-perceived aspects of wellbeing that are related to or affected by the presence of a disease or treatment [12]. A multidimensional HRQoL instrument was defined as any quality of life instrument assessing two or more of the three core domains described by the World Health Association: physical, social, and psychological wellbeing [13]. As a multidimensional construct, it includes perceptions, both positive and negative, of several dimensions such as physical, emotional, social and cognitive functioning. It also includes the negative aspects of somatization disorder and symptoms caused by a disease and/or its treatment [14]. Studies undertaken in different settings or in different countries might display slight divergences as HRQoL is also modulated by cultural and care patterns.

Over the past 20 years there has been a growing interest in the inclusion of HRQoL measures to assess the effects of a condition and/or its therapies on a person's health. In response to this interest, methods to assess health status and HRQoL have proliferated. There are now a 
number of valid and reliable instruments available for use in research investigations, which are the culmination of years of research with various populations, and reflect the target populations' perceptions of their health status and HRQoL [15].

HRQoL-measurement instruments validated for use in cancer patients have two basic categories. Questionnaires specifically designed for the disease explore the repercussions of the most typical symptoms and side-effects and are appropriate for comparing different treatment modalities or changes in patients. The general instruments are applicable to any population and are better suited to studies that seek to ascertain the disease's repercussion on HRQoL, taking the general population as reference [16]. Among the former, the most used in Europe for MM patients are the European Organization for Research and Treatment of Cancer Core Cancer Quality Life Questionnaire (EORTC QLQ-C30) and its MM-specific module (EORTC-QLQ-MY24/MY20). Among the latter, the Medical Outcomes Survey Short-Form General Health Survey (SF-36) is the most widely used.

There are also symptom-specific instruments, assessing the patient's reflections directly concerning pain, fatigue, neuropathy and nausea.

For example, the Functional Assessment of Chronic Illness Therapy (FACIT) system which is an established, comprehensive set of health-related quality-of-life measures includes a 27-item general measure, the Functional Assessment of Cancer Therapy (FACT-G), which can be combined with disease or treatment-specific subscales. The FACT-G captures four domains of health-related quality of life: physical, social, emotional and functional well-being. The supplemental subscales measure additional concerns of a specific disease or treatment. For example, the multiple myeloma subscale (FACT-MM) includes MM-relevant items There are also symptom-specific measures, such as FACT-An for patients with anemia or fatigue, FACTBone Pain: for patients with bone pain and treatment-specific measures assessing the QoL changing due to treatment such as FACT\&GOG-Ntx: for patients with neurotoxicity [17].

\section{Importance of quality of life issues}

In the case of MM, disease severity and type of treatment (high-dose chemotherapy and ASCT, the use of novel agents such as bortezomib, thalidomide or lenalidomide) have a clear influence on the patient's subjective perception of the disease. Their effects on HRQoL are also modulated by personality traits, personal resources and the availability and perception of social and family support.

Clinical applications of HRQoL tools may include prognostication, monitoring response to treatment, prioritizing problems or facilitating communication. The use of HRQoL instruments in clinical practice has also been shown to independently improve HRQoL in general oncology patients [18]. Some authors who have demonstrated reduced HRQoL in myeloma have concluded that HRQoL assessment should become a normal part of clinical care $[19,20]$.

Besides the typical primary parameters of clinical trials for measuring the treatment effect, such as tumor volume and time to progression, recognition of HRQoL is also an important 
endpoint in clinical research. In circumstances when the studied treatment modality results only a modest improvement in respect to primary parameters, with little benefit for the patient but with a significant side-effect profile, it may be a helpful outcome to detect the declination of HRQoL compared to the control. Clinical trials incorporating QoL assessments can provide more information and help clarify the relative harms and benefits of palliative chemotherapy and aid patient decisions when survival gains are small.

Delineation of side-effect profile by means of HRQoL assessment can assist in determining the types of supportive interventions that may be needed to ameliorate the side-effects.

QoL can also represent an independent prognostic factor. It is known that patients with a good QoL at the beginning of treatment manage better than those with a worse baseline value and there is a growing amount of evidences that QoL can be used as an effective prognostic indicator in respect to several kinds of malignancies [21].

QoL data can be a useful predictor of patient response to treatment and can affect decisionmaking about therapeutic options. This data allows patients to make informed and individualized decisions on the most appropriate treatment and any required supportive interventions.

HRQoL may be applied by the healthcare system to allocate resources by economic reality. As demand is always larger than resources, the optimal allocation of the financial means has great economic importance.

\section{Myeloma-specific HRQoL aspects}

$\mathrm{MM}$ is a chronic, incurable disease that is associated with reduced quality of life. MM patients have to face the problems of living with a chronic illness longer as a result of prolonged survival. However, they are also faced with the difficulties related to a malignant disease. Disease symptoms, concerns with certain therapeutic modalities and also the QoL changes due to organ transplantation emerge. Generation of pathologic bone fracture, bone pain, fatigue because of anemia and malignant disease itself, neurological symptoms due to hypercalcaemia have a profound impact on the QoL of MM patients.

Chronic renal failure develops in one third of $\mathrm{MM}$ patients. Chronic dialysis treatment implicates several life style changes.

Most anti-myeloma therapies involve intravenous injections or infusions. Regular laboratory check-ups require repeated blood sample collections that require multiple encounters with needles. Taking bone marrow for diagnosis and several times afterward for control examinations is very painful unless it is performed in narcosis.

ASCT has considerable effects on QoL. High-dose chemotherapy presents significant side effects and subsequently a reduction of QoL. This is due mainly to infections, mucositis, increased use of blood products and prolonged stays in the hospital. 
Side-effects of several lines of treatments include polyneuropathy, deep vein thrombosis, loss of hair and constipation. Osteonecrosis of the jaw caused by bisphosphonates, though a rare event, results in severe deterioration of QoL.

Recurrent infections due to the patients' immunocompromised status, the disease itself and also due to the several lines of treatment used to control the disease also contribute to the worsening of QoL.

\section{Questionnaires for evaluating MM}

Osborne et al. systematically reviewed the different HRQOL instruments applied for evaluating myeloma patients in their recent study. Thirteen different HRQOL instruments were identified across 39 studies. Only one disease-specific instrument was identified (EORTCQLQ-MY24/MY20). Other measures were general cancer tools (EORTC-QLQ-C30, FACT-An), treatment specific (EORTC-QLQ-HDC19, FACT-BMT), or generic [SF-36, SF-12, SEIQoL-DW, EQ-5D, 15D, life ingredient profile (LIP), Quality of Life Index (QLI)]. The SEIQoLDW was the only individualised instrument (with domains defined by respondents). No instrument was developed specifically for clinical use, or in palliative settings - although the search strategy was designed to identify these. [22].

No single instrument covered all issues identified as important by people with myeloma. The most comprehensive coverage was found in the EORTC-QLQ-MY24 (myeloma-specific module, used in conjunction with core cancer questionnaire EORTC-QLQ-C30), the FACTBMT and the QLI.

However, each tool has its strengths and the choice of tool will depend on the context in which it is used. To describe the incidence of side effects in a particular group, the EORTC tools may be more appropriate. However, in clinical practice, we may want a tool to focus more on the particular concerns of each patient (such as the SEIQoL-DW). These tools are time-consuming, require specialized training, are difficult to compare between studies and different interviewers and can be less feasible in certain groups such as those with chronic disease or the elderly.

Existing tools tend to be designed for use in research settings and their adaptation or the development of new tools specifically for use in clinical practice would be beneficial [22].

\section{HRQoL studies in MM}

Though HRQoL examinations are widely used especially in cancer patients and they are an integral component of clinical trials with new drugs, MM patients are relatively poorly studied in this respect. A PubMed search with terms of "multiple myeloma and quality of life or healthrelated quality of life" has resulted in only 51 items.

These studies targeted the comparison of HRQoL of MM patients in different countries [23], treated with different therapeutic schedules, receiving new drugs [24], underwent 
ASCT or tandem ASCT [25], special issues of the elderly [26], the effect of anemia and fatigue and also the effect of personality on disease outcome [27]. Methodological aspects are also emphasized [20].

\section{Disease-specific complaints and HRQoL of MM patients}

Patients with MM experience a very high symptom burden and low HRQOL. In a study published in 2012, the Eindhoven Cancer Registry was used to select all patients diagnosed with MM from 1999 to 2010. Patients were asked at baseline and 1 year later. Patients with MM reported statistically significant and clinically relevant worse scores on all EORTC QLQ-C30 scales compared to the norm. Also, patients with MM reported a mean decrease (e.g., worsening) between baseline and 1-year follow-up scores for: QoL $(74 \%$ of patients had a deteriorated score), fatigue (50\%), nausea and vomiting $(71 \%)$, pain $(59 \%)$ and dyspnoea $(66 \%)$. The most bothering symptoms during the past week were tingling hands/feet $(32 \%)$, back pain $(28 \%)$, bone aches/pain $(26 \%)$, pain in arm/shoulder $(19 \%)$ and feeling drowsy (18\%). Also, 37\% worried about their future health, 34\% thought about their disease and $21 \%$ worried about dying [28].

\section{QoL differences in transplant-ineligible myeloma patients treated with different drug combinations}

The phase 3 VISTA study (ClinicalTrials.gov NCT00111319) in transplant-ineligible myeloma patients demonstrated superior efficacy with bortezomib-melphalan-prednisone (VMP; nine 6-wk cycles) vs. melphalan-prednisone (MP) but also increased toxicity. HRQoL was evaluated using the EORTC-QLQ-C30 questionnaire. Results demonstrated clinically meaningful, transitory HRQoL decrements with VMP and relatively lower HRQoL vs. MP during early treatment cycles, associated with the expected additional toxicities. However, HRQoL is not compromised in the long term, recovering by the end-of-treatment visit to be comparable vs. MP. Analyses by bortezomib dose intensity indicated better HRQoL in patients receiving lower dose intensity [29].

\section{HRQoL assessment in MM patients undergoing autologous stem cell transplantation}

HRQoL assessment in this patient setting is important as patients and even clinicians are reluctant to choose this modality for fear of declination of QoL. However, it is not the best choice for every patient. HRQoL studies may contribute to the appropriate patient selection.

In a population-based study, the Nordic Myeloma Study Group found a survival advantage for high-dose therapy and ASCT compared to conventional chemotherapy in MM patients 
who were less than 60 years of age. HRQoL was integrated into the trial, using the EORTC QLQ-C30 questionnaire. Of the 274 patients receiving intensive therapy, 221 (81\%) were compared to 113 (94\%) of 120 patients receiving conventional melphalan-prednisone treatment. Prior to treatment, there were no statistically significant differences in any HRQoL score between the two groups. One month after the start of induction chemotherapy, the patients on intensive treatment had lower scores that gradually improved and at 12 and 24 months, the HRQoL was similar to that of the control patients. At 36 months, there was a trend toward less fatigue, pain, nausea, and appetite loss in the intensive-treatment group. Despite the moderate HRQoL reduction associated with the early intensive chemotherapy phase, the 18 months of prolonged survival seem to be associated with a good HRQoL [30].

QOL results of an Australian study on MM patients who underwent dose-reduced tandem ASCT were published in 2011. Patients younger than 60 years old received conditioning with melphalan $140 \mathrm{mg} / \mathrm{m}^{2}$ and patients who were Older than or equal to 60 years old received 100 $\mathrm{mg} / \mathrm{m}^{2}$. EORTC QLQ-C30 and the QLQ-MY24 questionnaires were conducted after each ASCT and thereafter every 3 months for 24 months. Mean global health measure improved from 3.44 before transplant to 4.50 (1being very poor and 7 being excellent) at the second and subsequent follow-up visits and the mean global QoL score improved from 3.61 to 4.71 . Pain symptoms were reduced and physical functioning improved throughout the period of post-transplant follow-up. The study showed that dose-reduced tandem ASCT was well tolerated with low toxicity although there was a transient reduction in QoL during both transplants. Posttransplant follow-up showed significant improvement in overall HRQoL that reflects positively on the overall disease-outcome [31].

In a University of Arkansas study, the decreases in functioning after transplantation were less pronounced than anticipated. At stem cell collection, physical deficits were common, with most patients scoring 1 standard deviation below population norms for physical well-being $(70.2 \%)$ and functional well-being $(57.5 \%)$, and many reporting at least moderate fatigue $(94.7 \%)$ and pain $(39.4 \%)$. Clinically meaningful levels of anxiety $(39.4 \%)$, depression $(40.4 \%)$ and cancer-related distress $(37.0 \%)$ were evident in a notable proportion of patients. After transplantation, there was a worsening of transplant-related concerns, depression and lifesatisfaction. However, pain improved and social functioning was well preserved. Older patients were not more compromised than younger ones. In multivariate analyses, they reported better overall QoL and less depression than before transplantation [32].

\section{QoL assessment of elderly MM patients}

Thalidomide with melphalan and prednisone (MPT) was defined as standard treatment in elderly patients with MM. In a randomized trial (HOVON49), a prospective HRQoL study was initiated in order to assess the impact of thalidomide on QoL. Patients aged 65 years and older with newly diagnosed MM were randomized to receive melphalan plus prednisone (MP) or MPT, followed by thalidomide maintenance in the MPT arm. 284 patients were included (MP, n=149; MPT n=135). HRQoL was assessed with the QLQ- 
C30 and the myeloma-specific module (QLQ-MY24) at baseline and at predetermined intervals during treatment. The QLQ-C30 subscales physical function and constipation showed an improvement during induction in favour of the MP arm. During thalidomide maintenance, the scores for the QLQ-MY24 paraesthesia became significantly higher in the MPT arm. The QLQ-C30 subscales pain, insomnia and appetite loss and the QLQ-MY24 item sick scored marginally better during thalidomide maintenance. The overall QoLscale QLQ-C30-HRQoL showed a significant time trend towards more favorable mean values during protocol treatment without differences between MP and MPT. For the QLQC30 subscales emotional function and future perspectives, difference in favour of the MPT arm from the start of treatment was observed with no significant 'time $\times$ arm' interaction, indicating a persistent better patient perspective with MPT treatment. The study concluded that the higher frequency of toxicity associated with MPT does not translate into a negative effect on HRQoL and that MPT holds a better patient perspective [33].

Quality-of-life assessment may be an independent and valuable addition to the known prognostic factors in multiple myeloma. In a randomized trial (NMSG 4/90), patients treated with melphalan/prednisone were compared to a melphalan/prednisone + interferon alpha- $2 \mathrm{~b}$ treated patient group in 486 newly diagnosed multiple myeloma. Univariate analysis showed a highly significant association with survival from the start of therapy for physical functioning as well as role and cognitive functioning, global quality of life, fatigue and pain. In multivariate analysis, physical functioning and W.H.O. performance status were independent prognostic factors when analysed in a Cox regression model with the somatic variables beta- 2 microglobulin, skeletal disease and age. The best prediction for survival from the start of therapy was obtained by combining the beta- 2 microglobulin and physical functioning scores in a variable consisting of three risk factor levels with an estimated median survival of 17, 29 and 49 months, respectively [34].

\section{Assessment of the correlation of psychological well-being and QoL in MM}

A cross-sectional survey was conducted aiming to identify the nature and range of needs, as well as levels of quality of life (QoL), of both patients living with myeloma and their partners. Patients and their partners were recruited from 4 hospitals in the United Kingdom at a mean post-diagnosis time of 5 years. A total of 132 patients and 93 of their partners participated. One-quarter of the patients and one-third of the partners reported unmet supportive care needs. About $27.4 \%$ of patients reported signs of anxiety and $25.2 \%$ reported signs of depression. Almost half the partners $(48.8 \%)$ reported signs of anxiety and $13.6 \%$ exhibited signs of depression. Anxious/depressed patients had more than double the unmet needs than non-anxious/depressed patients $(\mathrm{P}<0.05)$. QoL was moderate, with key areas of impairment being physical, emotional, social and cognitive functioning. Patients complained of several symptoms, including tiredness $(40.7 \%)$, pain $(35.9 \%)$, insomnia $(32.3 \%)$, peripheral neuropathies $(28.3 \%)$ and memory problems $(22.3 \%)$. About $40.8 \%$ were worried about their health in the future [35]. 


\section{Conclusion}

Investigation of QoL has become increasingly important in economically developed countries. HRQoL assessment is becoming a current and integral part of clinical studies with new drugs. Measuring of QoL is becoming more and more important for decision making in the field of health policy.

$\mathrm{MM}$ is a currently incurable disease, but survival can be significantly prolonged by the administration of new therapeutic modalities. The mean age at the time of diagnosis is over 60 , so it is especially important to choose the least harmful treatment for the patient so the best quality of life can be achieved. Results of QoL examinations can help us find the most appropriate treatment for our patients.

\section{Abbreviations}

ASCT: autologous stem cell transplantation; EORTC: European Organization for Research and Treatment of Cancer; HRQoL: health-related quality of life; Ig: immunoglobulin; MM: multiple myeloma; QoL: quality of life; WHO: World Health Organization

\section{Author details}

Klára Gadó* and Gyula Domján

*Address all correspondence to: gadok@freemail.hu

Semmelweis University, Faculty of Medicine, $1^{\text {st }}$ Department of Internal Medicine, Budapest, Hungary

\section{References}

[1] Ferlay, J, Shin, H. R, Bray, F, Forman, D, Mathers, C, \& Parkin, D. M. Estimates of worldwide burden of cancer in (2008). GLOBOCAN 2008. Int J Cancer. 2010;, 127, 2893-917.

[2] Raab, M. S, Podar, K, Breitkreutz, I, Richardson, P. G, \& Anderson, K. C. Multiple myeloma. Lancet. (2009). , 374(9686), 324-39.

[3] Sirohi, B, \& Powles, R. Epidemiology and outcomes research for MGUS, myeloma and amyloidosis. Eur J Cancer. (2006). , 42, 1671-83. 
[4] Cherry, B. M, Korde, N, Kwok, M, Roschewski, M, \& Landgren, O. Evolving therapeutic paradigms for multiple myeloma: back to the future. Leuk Lymphoma. (2012). doi:10.3109/10428194.2012.717277)

[5] Greipp, P. R. San Miguel J, Durie BG, et al. International staging system for multiple myeloma. J Clin Oncol. (2005). , 23, 3412-20.

[6] Attal, M, Harousseau, J. L, Stoppa, A. M, Sotto, J. J, et al. A prospective randomized trial of Autologous Bone Marrow Transplantation and chemotherapy in Multiple Myeloma. N Engl J Med. (1996). , 335, 91-97.

[7] Wildes, T. M, Vij, R, Petersdorf, S. H, Medeiros, B. C, \& Hurria, A. New Treatment Approaches for Older Adults with Multiple Myeloma. J Geriatr Oncol. (2012). Epub 2012 Feb 28., 3(3), 279-290.

[8] Kyle, R. A, \& Rajkumar, S. V. Criteria for diagnosis, staging, risk stratification and response assessment of multiple myeloma. Leukemia. (2009). , 23, 3-9.

[9] Attal, M, \& Olivier, P. Cances Lauwers V, et al. Maintenance treatment with lenalidomide after transplantation for myeloma. Analysis of secondary malignancies within the IFM trial. Haematologica (2011). suppl 1):s23., 2005-02.

[10] Richardson, P. G, Laubach, J. P, Schlossman, R. L, Ghobrial, I. M, Mitsiades, C. S, Rosenblatt, J, Mahindra, A, Raje, N, Munshi, N, \& Anderson, K. C. The Medical Research Council Myeloma IX trial: the impact on treatment paradigms. Eur J Haematol. (2012). , 88(1), 1-7.

[11] Kuyken, W, Orley, J, Hudelson, P, \& Sartorius, N. Quality of life assessment across cultures. Int. J. Mental Hlth, 23, 5. (WHOQOL): position paper from the World Health Organization. Soc. Sci. Med., (1994).

[12] Ebrahim, S. Clinical and Public-Health Perspectives and Applications of Health-Related Quality-Of-Life Measurement. Soc Sci Med. (1995). doi:O., 41, 1383-1394.

[13] WHOQOL-GroupThe World Health Organization quality of life assessment (WHOQOL): Position paper from the World Health Organization. Soc Sci Med. (1995). , 41(10), 1403-9.

[14] Osoba, D. Lessons Learned from Measuring Health-Related Quality-Of-Life in Oncology. J Clin Oncol. (1994). , 12, 608-616.

[15] Naughton, M. J, \& Shumaker, S. A. The case for domains of function in quality of life assessment. Qual Life Res. (2003). Suppl , 1, 73-80.

[16] Ferrans, C. E. In: Outcomes Assessment in Cancer. Measures, Methods, and Applications. 1. Lipscomb J, Gotay CC, Snyder C, editor. Cambridge: Cambridge University Press; (2005). Definitions and conceptual models of quality of life; , 14-30. 
[17] Webster, K, Cella, D, \& Yost, K. The Functional Assessment of Chronic Illness Therapy (FACIT) measurement system: properties, application, and interpretation. Health Qual Life Outcomes. (2003). , 1, 79-86.

[18] Velikova, G, Booth, L, Smith, A. B, Brown, P. M, Lynch, P, Brown, J. M, et al. Measuring Quality of Life in Routine Oncology Practice Improves Communication and Patient Well-Being: A Randomized Controlled Trial. J Clin Oncol. (2004). , 22(4), 714-24.

[19] Sherman, A. C, Simonton, S, Latif, U, Plante, T. G, \& Anaissie, E. J. Changes in quality-of-life and psychosocial adjustment among multiple myeloma patients treated with high-dose melphalan and autologous stem cell transplantation. Biol Blood Marrow Transplant. (2009). , 15(1), 12-20.

[20] Osborne, T. R, Ramsenthaler, C, Siegert, R. J, Edmonds, P. M, Schey, S. A, \& Higginson, I. J. What issues matter most to people with multiple myeloma and how well are we measuring them? A systematic review of quality of life tools. Eur J Haematol. (2012). Sep 18. doi:ejh.12012.

[21] Fallowfield, L. Quality of life: a new perspective for cancer patients. Nat Rev Cancer. (2002). , 2(11), 873-9.

[22] Osborne, T. R, et al. What issues matter most to people with multiple myeloma and how well are we measuring them? A systematic review of quality of life tools. Eur J Haematol. (2012). Sep 18. doi:ejh.12012.

[23] Kontodimopoulos, N, Samartzis, A, Papadopoulos, A. A, \& Niakas, D. Reliability and Validity of the Greek QLQ-C30 and QLQ-MY20 for Measuring Quality of Life in Patients with Multiple Myeloma. ScientificWorldJournal. (2012).

[24] Alegre, A, Oriol-rocafiguera, A, Garcia-larana, J, Mateos, M. V, Sureda, A, Martinezchamorro, C, Cibeira, M. T, Aguado, B, Knight, R, \& Rosettani, B. Efficacy, safety and quality-of-life associated with lenalidomide plus dexamethasone for the treatment of relapsed or refractory multiple myeloma: the Spanish experience. Leuk Lymphoma. (2012). Epub 2012 Mar 1., 53(9), 1714-21.

[25] Naumann-winter, F, Greb, A, Borchmann, P, Bohlius, J, Engert, A, \& Schnell, R. Firstline tandem high-dose chemotherapy and autologous stem cell transplantation versus single high-dose chemotherapy and autologous stem cell transplantation in multiple myeloma, a systematic review of controlled studies. Cochrane Database Syst Rev. (2012). Oct 17;10:CD004626. doi:CD004626.pub3.

[26] Verelst, S. G, \& Termorshuizen, F. Uyl-de Groot CA, Schaafsma MR, Ammerlaan AH, Wittebol S, Sinnige HA, Zweegman S, van Marwijk Kooy M, van der Griend R, Lokhorst HM, Sonneveld P, Wijermans PW; Dutch-Belgium Hemato-Oncology Cooperative Group (HOVON). Effect of thalidomide with melphalan and prednisone on health-related quality of life (HRQoL) in elderly patients with newly diagnosed multiple myeloma: a prospective analysis in a randomized trial. Ann Hematol. (2011). Epub 2011 Apr 7., 90(12), 1427-39. 
[27] Strasser-weippl, K, \& Ludwig, H. Psychosocial QOL is an independent predictor of overall survival in newly diagnosed patients with multiple myeloma. Eur J Haematol. (2008). Epub 2008 Jul 11, 81(5), 374-9.

[28] Mols, F, Oerlemans, S, Vos, A. H, Koster, A, Verelst, S, \& Sonneveld, P. van de PollFranse LV. Health-related quality of life and disease-specific complaints among multiple myeloma patients up to $10 \mathrm{yr}$ after diagnosis: results from a population-based study using the PROFILES registry. Eur J Haematol. (2012). doi:j. 1600-0609.2012.01831.x. Epub 2012 Aug 1, 89(4), 311-9.

[29] Delforge, M, \& Dhawan, R. Robinson D Jr, Meunier J, Regnault A, Esseltine DL, Cakana A, van de Velde H, Richardson PG, San Miguel JF. Health-related quality of life in elderly, newly diagnosed multiple myeloma patients treated with VMP vs. MP: results from the VISTA trial. Eur J Haematol. (2012). doi:j.1600-0609.2012.01788.x. Epub 2012 May 7., 89(1), 16-27.

[30] Gulbrandsen, N, Wisløff, F, Brinch, L, Carlson, K, Dahl, I. M, Gimsing, P, Hippe, E, Hjorth, M, Knudsen, L. M, Lamvik, J, Lenhoff, S, Løfvenberg, E, Nesthus, I, Nielsen, J. L, \& Turesson, I. Westin J; Nordic Myeloma Study Group. Health-related quality of life in multiple myeloma patients receiving high-dose chemotherapy with autologous blood stem-cell support. Med Oncol. (2001). , 18(1), 65-77.

[31] Khalafallah, A, Mcdonnell, K, Dawar, H. U, et al. Quality of life assessment in multiple myeloma patients undergoing dose-reduced tandem autologous stem cell transplantation. Mediterr J Hematol Infect Dis. (2011). e2011057. Epub 2011 Nov 28.

[32] Sherman, A. C, Simonton, S, Latif, U, Plante, T. G, \& Anaissie, E. J. Changes in quality-of-life and psychosocial adjustment among multiple myeloma patients treated with high-dose melphalan and autologous stem cell transplantation. Biol Blood Marrow Transplant. (2009). , 15(1), 12-20.

[33] Verelst, S. G, \& Termorshuizen, F. Uyl-de Groot CA, Schaafsma MR, Ammerlaan AH, Wittebol S, Sinnige HA, Zweegman S, van Marwijk Kooy M, van der Griend R, Lokhorst HM, Sonneveld P, Wijermans PW; Dutch-Belgium Hemato-Oncology Cooperative Group (HOVON). Effect of thalidomide with melphalan and prednisone on health-related quality of life (HRQoL) in elderly patients with newly diagnosed multiple myeloma: a prospective analysis in a randomized trial. Ann Hematol. (2011). Epub 2011 Apr 7., 90(12), 1427-39.

[34] Wisløff, F, \& Hjorth, M. Health-related quality of life assessed before and during chemotherapy predicts for survival in multiple myeloma. Nordic Myeloma Study Group. Br J Haematol. (1997). , 97(1), 29-37.

[35] Molassiotis, A, Wilson, B, Blair, S, Howe, T, \& Cavet, J. Unmet supportive care needs, psychological well-being and quality of life in patients living with multiple myeloma and their partners. Psychooncology. (2011). , 20(1), 88-97. 